\title{
Research on Automatic Generation of Table Tennis Technique and Tactics Collection Template
}

\author{
Jing Sun, Haochen Luo, and Huiqun Zhao
}

\begin{abstract}
In the professional table tennis team, the content of the game is summarized after each contest. The summary process needs to set up technical and tactical acquisition templates according to the coach's intention, and then perform technical and tactical data collection and analysis according to the template. However, due to the randomness of the template, manual design of the template requires a lot of manpower and time, making the technical and tactical collection efficiency very low. In order to improve the acquisition speed, this paper analyzes the recommendation algorithm based on collaborative filtering and its implementation process. Combined with the process of table tennis technology and tactics collection and analysis, this paper proposes a solution to automatically generate table tennis tactics acquisition template. Experiments show that this method reduces the time required by the method of manually designing the template by $50 \%$ and improves the collection efficiency.
\end{abstract}

Index Terms-Table tennis skills and tactics collection, recommendation system, coll, orative filtering, automated generation.

\section{INTRODUCTION}

In recent years, the technical and tactical analysis of computer technology applied to sports competitions has been commonplace [1]. This year I participated in the technical and tactical collection and analysis of the national table tennis team. In the field of tactics analysis of table tennis, coaches need to design technical and tactical acquisition templates before collection for the purpose of researching the skills and tactics used by athletes in the competition [2]. The template content consists of detailed technical and tactical indicators such as player teeing skills, receiving teeing techniques, rotation conditions, means information, and gains and losses. Each time before the collection, the technicians will select the technical and tactical content that matches the collection intention according to the coach's collection intention, and form a set of acquisition templates. Each collection template is a technical and tactical system. With the increase of game data and the continuous enrichment of technical and tactical data, how to quickly establish targeted technical and tactical templates has become a major problem for coaches.

In the daily training of the current national team, the coaches used a method of re-establishing templates for different games to solve this problem. However, the technical

Manuscript received March 12, 2019; revised December 13, 2020. This work was supported in part by Natural Science Foundation of China (Grant No: 61672041, 61370051). It is also partly supported by Beijing Government and Education Committee (Grant No. PHR201107107).

The authors are with Computer School, North China University of Technology, Beijing, 100144, China (e-mail: sunjing8248@163.com, 923816437@.com, zhaohq6625@sina.com). and tactical template needs to be manually established by the technician according to the coach's intention. Re-establishing the template before each collection wastes a lot of time and effort of the technician.

In order to reduce the template design time and improve the work efficiency, this paper proposes a solution to automatically generate templates in the design of table tennis tactics template using collaborative filtering. Recommendation algorithms include content-based recommendations, combined recommendations, and recommendations based on collaborative filtering [3]. Among them, collaborative filtering algorithm is the most successful algorithm in the field of personalized recommendation, which has simple and efficient features [4]. The technology can be based on the technical and tactical requirements of the coaches and the historical data that has been used, combined with the characteristics of the technical and tactical templates, to build a personalized recommendation algorithm, provide personalized recommendation services, and provide targeted technical and tactics for the coaches. Templates to improve the efficiency of technical and tactical analysis.

This paper mainly introduces how the automatic generation of table tennis technical and tactical templates is realized. Section 2 mainly introduces related research; Section 3 mainly introduces the algorithm design flow of table tennis tactics recommendation system based on collaborative filtering; Section 4 mainly introduces related experiments and results analysis; and finally concludes.

\section{DESIGN OF RECOMMENDATION ALGORITHM BASED ON COLLABORATIVE FILTERING}

Since the similarity between the user and the user cannot be measured in the table tennis template recommendation, and each template cannot be measured by a quantified score table, such as cosine similarity (COS), Pearson correlation coefficient (PCC) other user-based recommendations and project-related recommendations are not well suited for table tennis tactical templates. So here we have chosen model-based collaborative filtering. We use association algorithms for collaborative filtering. Only need to provide relevant historical data used in the past for analysis, you can make the corresponding template recommendation. This is a good way to avoid the need for a quantified score sheet. Based on this, we also combine semantic keyword matching. The coaches intend to process the word segmentation and find the technical and tactical keywords, and combine these keywords with the optimal history template obtained through the collaborative filtering algorithm to form a new targeted template and recommend it to the coach. 
The implementation process of the technical and tactical recommendation system based on the collaborative filtering recommendation algorithm mainly includes four steps. The first is to collect historical technical and tactical data, and form a historical technical and tactical template data set based on the collected data. Secondly, according to the collected data set, the fp-tree algorithm is used to perform frequent data set mining, and a frequent set sequence satisfying the support threshold is found. Then, the collection intention provided by the coach is processed by word segmentation, and the keywords are compared with the technical and tactical content in the frequent episode sequence, and the frequent itemsets overlapping with the intention are selected. Finally, according to certain scoring standards, frequent items are reorganized, and corresponding technical and tactical templates are formed and recommended to the coaches for use. Fig. 1 shows the relevant flow chart of the system.

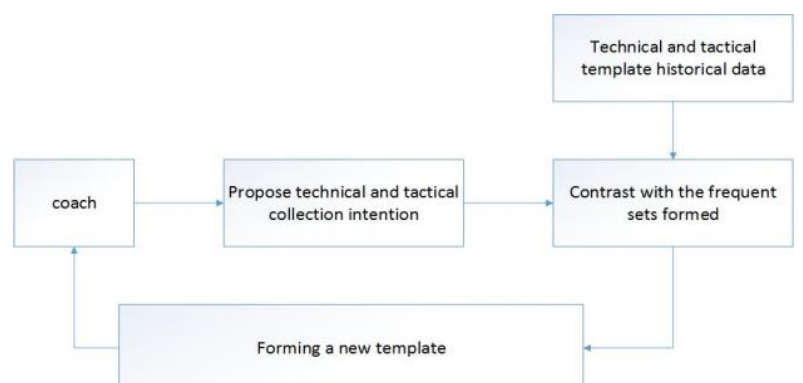

Fig.1. Flow chart of recommendation system based on collaborative filtering.

\section{A. Historical Technical and Tactical Data Preprocessing}

The historical data required for the table tennis technical and tactical template recommendation, including the technical and tactical templates used in the past, the technical and tactical details that need to be collected, the specific technical and tactical information, and the collection intentions used in the past. These basic data are derived from the pre-processing of the templates used to collect data from previous games. These basic data will increase based on the increase in analysis sessions. The analysis process performed later is based on the data obtained from the preprocessing. The specific data is shown in Table I.

TABLE I: TABLE TENNIS TACtics Template DATA SheET

\begin{tabular}{ll}
\hline \hline Number & Template data \\
\hline 1 & $\begin{array}{l}\text { Number of plates, Means, Placement, Winning and losing } \\
\text { points }\end{array}$ \\
\hline 2 & Means, Placement, Winning and losing points \\
\hline 3 & $\begin{array}{l}\text { Serve and catch ball, Number of plates, Placement, } \\
\text { Winning and losing points }\end{array}$ \\
\hline 4 & Serve technology, Means, Winning and losing points \\
\hline 5 & $\begin{array}{l}\text { Catching technology, Number of plates, Winning and } \\
\text { losing points }\end{array}$ \\
\hline$\cdots \cdots$ & $\cdots \cdots$ \\
\hline \hline
\end{tabular}

\section{B. Mining Data Frequent Sets Using FP-Tree Algorithm}

The strategy of the FP-tree algorithm is to use a divide-and-conquer method to scan the database first, and then generate a frequent pattern tree (FP-tree) according to the associated frequency set. Then it is divided into several conditional libraries, each of which is related to the frequency set of length 1. Finally, the specific conditional library is excavated separately. Here we first set the minimum support to 3 , because of the particularity of table tennis skills and tactics, many techniques and tactics occur rarely because the technical and tactical is more targeted than because they appear meaningless, so support Set to 3 , to ensure that the targeted techniques and tactics appear, can also avoid those basic tactics and tactics that have not appeared as interference items are recommended. Then we use the fp-tree algorithm for data mining of the table tennis technical and tactical template data that was originally summarized. Before you start mining, you must first build an fp-tree. The first scan of the pre-processed historical technical and tactical data is performed, and the frequency of each technical tactic is extracted, and the head table is formed by the frequency. Then, the item header table is arranged in descending order, and the items whose frequency is less than the support threshold are eliminated. Then, according to the item header table arranged in descending order, the items in the original transaction set smaller than the support threshold are deleted, and the remaining items are arranged in the order of the item header table. Then scan the transaction set a second time, insert the preprocessed transaction into the constructed fp-tree, each technique and tactic will be used as a leaf node of the formed fp-tree, and all transactions will be inserted into the tree in turn. After that, you can form a complete fp-tree. After generating an fp-tree, you can generate a frequent item set for the node by selecting any node in the item header table and then traversing up to the root node of the tree. Each frequent item set is a combination of skill and tactics; the frequency of each frequent item set is recorded. These frequencies are the number of times of use of these technical and tactical combinations; these data are supported as data for the subsequent frequent item set reorganization into templates. Part of the excavation situation is shown in Table II.

TABLE II: FREQUENT ITEMSETS FREQUENCY TABLE

\begin{tabular}{ll}
\hline \hline Frequent itemsets & Frequency \\
\hline Winning and losing points, Number of plates & 6 \\
\hline $\begin{array}{l}\text { Number of plates, Serve and catch ball, Winning and } \\
\text { losing points, Rotate }\end{array}$ & 5 \\
\hline Serve and catch ball, Number of plates & 13 \\
\hline Winning and losing points, Means, Number of plates & 10 \\
\hline $\begin{array}{l}\text { Rotate, Number of plates, Serve and catch ball, Serve and } \\
\text { catch technology }\end{array}$ & 3 \\
\hline$\cdots \cdots$ & $\cdots \cdots$ \\
\hline \hline
\end{tabular}

The fp-tree algorithm is constructed as follows:

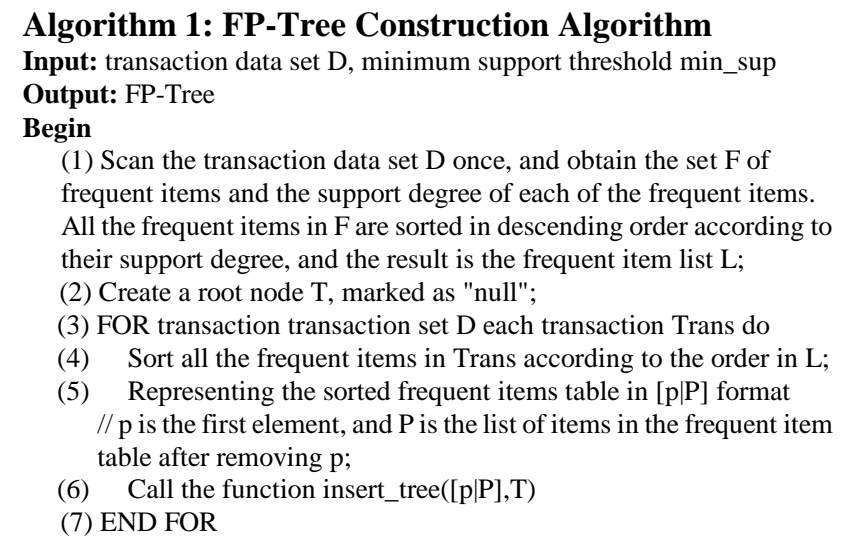

Input: transaction data set D, minimum support threshold min_sup Output: FP-Tree

(1) Scan the transaction data set D once, and obtain the set F of frequent items and the support degree of each of the frequent items. All the frequent items in $\mathrm{F}$ are sorted in descending order according to

(4) Sort all the frequent items in Trans according to the order in L;

5) Representing the sorted frequent items table in $[\mathrm{p}[\mathrm{P}]$ format table after removing $\mathrm{p}$;

(7) END FOR 


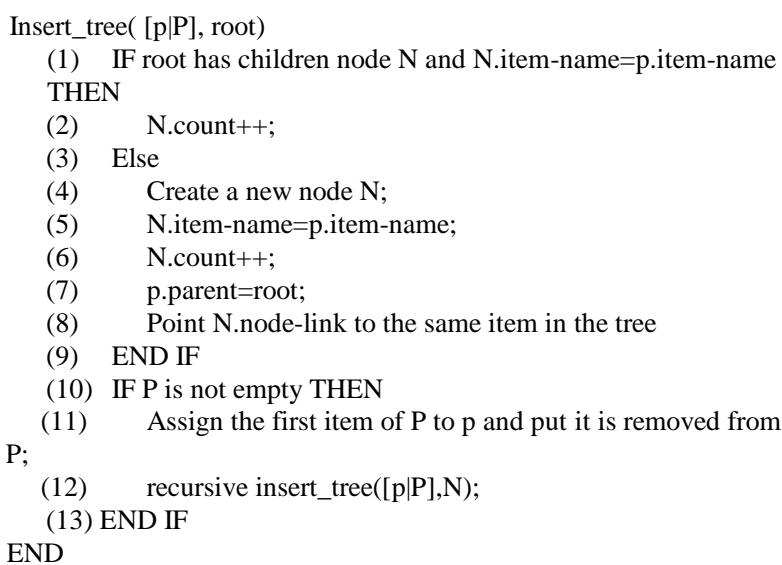

The algorithm for extracting frequent sets from fp-tree is as follows:

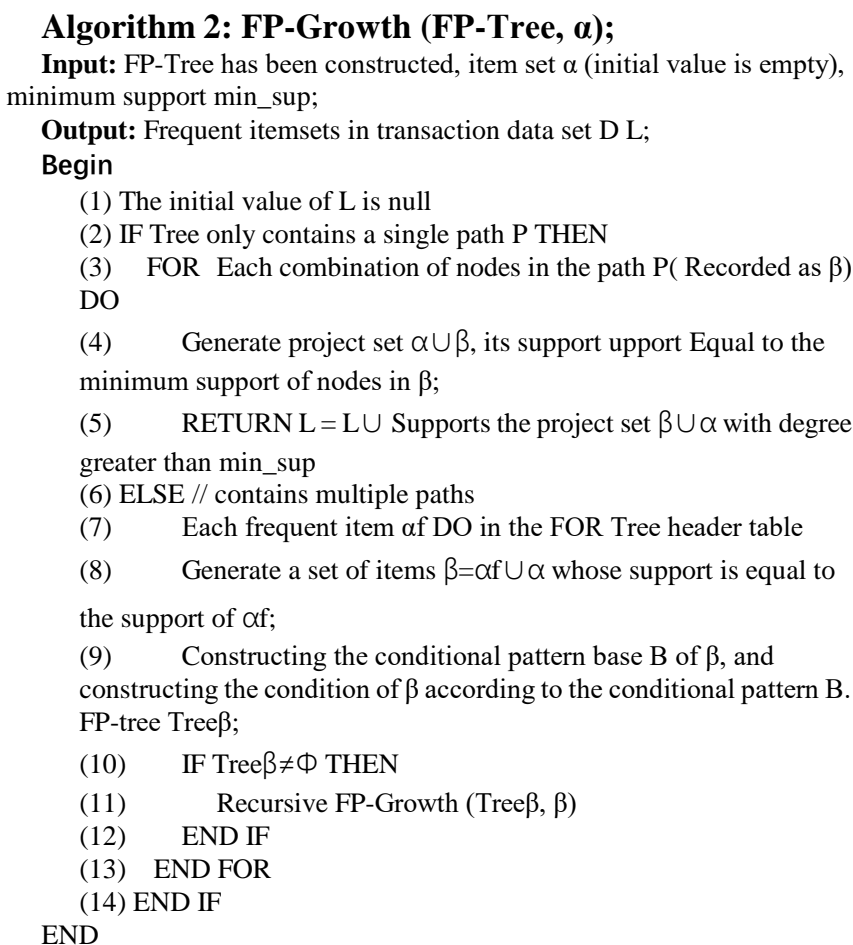

\section{Semantic Participle Matching Algorithm}

Since the coaches' intentions are often one sentence, it is usually impossible to find out the technical and tactical content that the coaches really need to collect based on one sentence. Therefore, the strategy we adopt is to first classify the coach's intentions, and then compare the separated words with the existing frequent items to select frequent items that match the coach's intention. Here we use the forward maximum matching algorithm for word segmentation. The word segmentation dictionary we used has updated a lot of tactics related to table tennis, and will update the word segmentation dictionary according to the coach's needs. This will ensure that the skills and tactics needed to be collected are completely matched, and no missing key words will be found. Happening. At the same time, each time the coach will record the intention of the collection, so that the coach can directly select the used intentions to use directly or slightly modify the collection, and collect the analysis techniques and tactics for the coaches. Save time and increase efficiency. The forward maximum matching algorithm used is as follows:

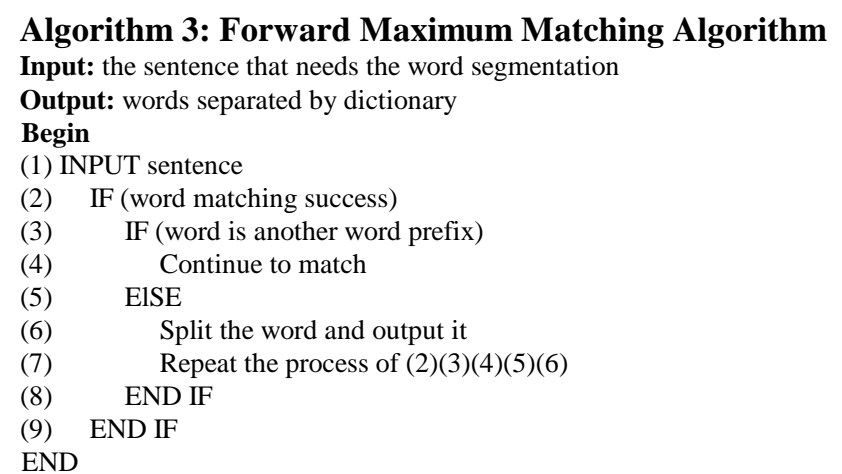

\section{Recommend Based on Frequent Episodes}

Firstly, according to the result of the word segmentation, the table tennis technical and tactical content appearing in the coach's intention is recorded first. These contents are the basic intentions of the coaches, that is, the semantic keywords that need to be matched later. Since these keywords are the requirements of the coaches this time, these keywords must appear in the recommended template. These keywords are then matched against frequent itemsets extracted from historical data. All matching frequent itemsets are now selected and their conditional probabilities are calculated and recorded. Then, according to the size of the conditional probability, the most template supplement with the highest conditional probability is selected. Reorganize the keywords proposed by the coaches and the selected frequent itemsets to form a new template, and recommend this new template. The specific flow chart is shown in Fig. 2.

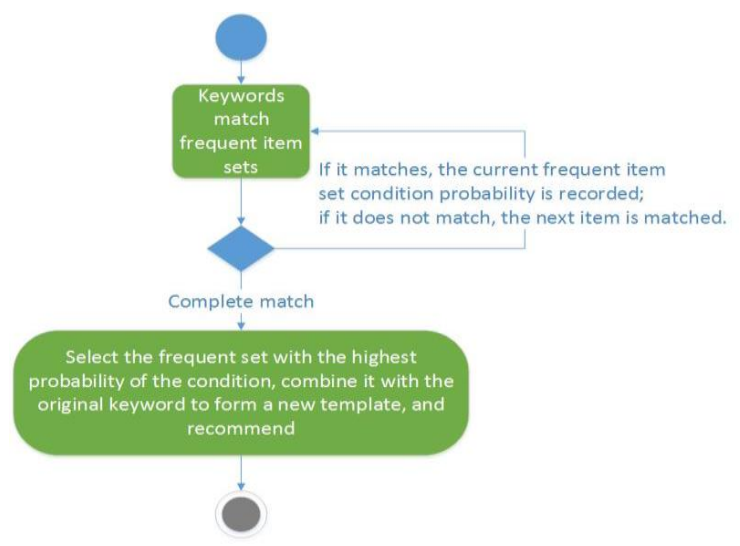

Fig. 2. Recommended process

\section{EXPERIMENT AND RESULT ANALYSIS}

We used vs 2015 in the memory $8 \mathrm{~g}$, cpu for Intel (R) Core (TM) i5-3230M, the operating system is windows 7 on the computer to achieve the system. The following describes the data used and the analysis of the results.

\section{A. Data Used in the Experiment}

The data set we used was derived from the real training data of the national table tennis team. I selected five of the game data as an example for experimentation. The first game is Malone vs Boer. The coaches collected the intention to collect the Malone forehand teeing skills and the points of gain and loss. The second is Xu Wei vs. Fan Zhendong. The coaches collected the intention to collect the $\mathrm{Xu}$ Wei serve and the rotation of the serve. It is Malone vs Ochalov. The 
coaches collect the intentions to collect the means of serving the Malone, the means of receiving the ball and the points of gain and loss. The fourth is Fan Zhendong vs. Shuiguyu. The coach collects the intention to collect Fan Zhendong's means of losing points. Number, the number of boards held; the fifth is Fan Zhendong vs Ma Long, the coach's collection intention is to collect Fan Zhendong's teeing skills, pick up the ball technology, drop the situation and the points of gain and loss.

\section{B. Experiment and Result Analysis}

In order to evaluate the quality of the template generated automatically, we use the method of this paper to compare the traditional methods of manually creating templates.

The template items manually created in the game one have bureau, ratio, minute, backhand, haircut, teeing technique, and score. The recommended templates are bureau, ratio, minute, pros and cons, send and receive, serve skills, gain and loss points, Number of boards.

The template items manually created in the game two have bureau, ratio, minute, send, tee, technique, and rotation; the recommended templates are bureau, ratio, minute, send, means, rotation, gain and loss points.

The template items manually created in the game three have bureau, ratio, point, send and receive, serve technology, receive the ball technology, means, gains and losses points; the recommended templates are bureau, ratio, point, send, means, gains and losses, number of boards.

The template items manually created in the game four have bureau, ratio, score, score, means, number of boards, and number of boards; the recommended templates are bureau, ratio, point, gain and loss points, means, number of boards, and number of boards.

The template items manually created in the game five have ratio, point, send, send technology, receive the ball technology, means, drop points, gains and losses points; recommended templates for the bureau, ratio, points, send, serve, receive the ball Technology, drop point, gains and losses, number of boards.

\section{(1) Comparison of time used}

As can be seen from Fig. 3, the time required to manually create a template is about $50 \%$ longer than the time required to recommend the template. Therefore, the use of the template recommendation system can save more acquisition time, and can save manpower and improve work efficiency.

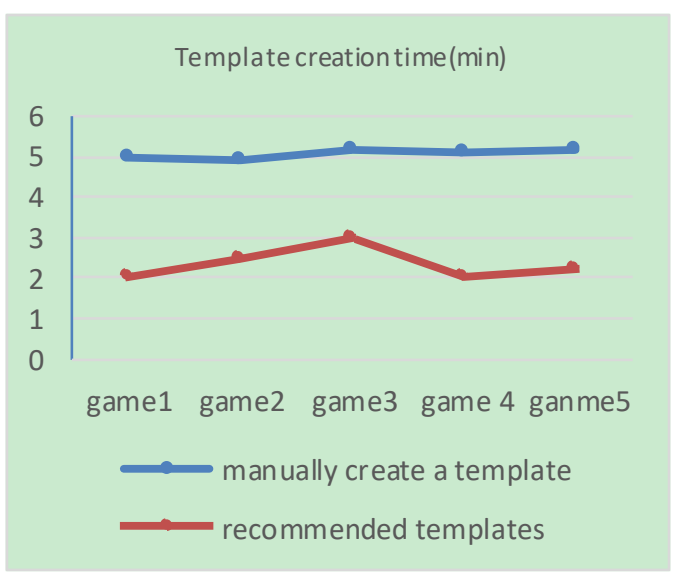

Fig. 1. Template creation time comparison chart.

(2) Accuracy comparison
We use the manually created template items as the standard, and the accuracy of each template recommendation is shown in Fig. 4. It can be seen that if the template item has a character difference from the coach's intention, it will cause the keyword to be mismatched with the frequent item set, resulting in the recommended template accuracy rate is not very high, but if the template item matches the coach's intention Keywords and frequent itemsets can be perfectly matched, and the recommended accuracy rate can reach $100 \%$.

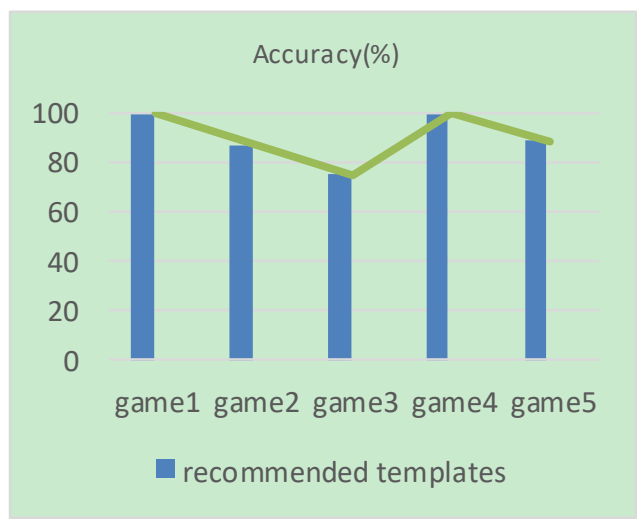

Fig. 4. Recommended template accuracy graph.

(3) Comprehensive analysis

According to the comprehensive analysis of Figure 3 and Figure 4, if the coach's intention is close to the template item and the recommendation accuracy is high, the time taken to establish the template can be greatly reduced, and the work efficiency is improved. However, when the coach's intention is different from the template item and the accuracy cannot be guaranteed, the technician needs to modify it according to the recommended template, which will lead to an increase in the required time, but still more than the manual establishment of the template. The short time required can increase work efficiency slightly. Some Common Mistakes

\section{RELATED WORK}

The personalized recommendation system was originally applied in the commercial field, mainly in the recommendation of purchasing items, the recommendation of music, the recommendation of browsing news, etc. [5]. In these areas, the recommendation system has achieved significant success. Now, the recommendation system has been applied to various fields. For example, Jing Wang, Chuntao Man and others applied the recommendation system to the medical community question and answer system, which facilitated the communication between patients and doctors [6]; the online book recommendation system designed by Nursultan Kurmashov et al. can help readers find fast and accurate. Books suitable for them to read [7]; Hualun Ma and others apply the recommendation system to the course recommendation, so that students can choose the course they are satisfied with [8]; the comic recommendation system designed by Sun, Y, T. Wang and others can Everyone is expected to recommend the preference for unread comics [9]. Faustino Sanchez, María Alduan, Federico Alvarez et al. 
designed a sports video recommendation system that uses the information available in the video to recommend users. The system has been tested in the Olympics [10]. Others have optimized and improved the collaborative recommendation algorithm. For example, Anand Kishor Pandey and others have optimized the cold start problem of collaborative filtering by studying the similarity between new and old users [11]. Shiping Fan and others have improved the reliability of user similarity calculation by introducing the credibility between users [12].

The table tennis technical and tactical template is widely used in the analysis of table tennis skills and tactics. Zhang Xiaodong, Xiao Dandan and others proposed the three-segment statistical method of table tennis techniques and tactics [13], and Wu Fei, Liu Guobing and others proposed to improve the three-segment technique and tactics of table tennis [14] First set up technical and tactical templates for technical and tactical analysis after data extraction.

Although the application of personalized recommendation service is very wide, its application in the field of table tennis techniques and tactics is still in the research and exploration stage. Therefore, it is necessary to study the application of personalized recommendation service in the collection of table tennis skills and tactics. The core is to study the recommendation service of table tennis technical and tactical templates. Recommending a good template can greatly reduce the coach's repetitive work, save a lot of time and improve work efficiency. The recommended system in the collection of table tennis skills and tactics includes different players, different technical and tactical content and different coaches' needs. On this basis, the matching recommendation algorithm is selected to optimize the recommendation process, which can be accurate and fast. Recommend the table tennis technical and tactical template to the coach.

\section{CONCLUSION}

Through experiments, we can see that the technical and tactical analysis through the recommendation system will save $50 \%$ of the time by manual completion, which can improve the efficiency of the coach analysis game. However, we can also find that when the template item is different from the coach's intention, the recommendation system cannot perfect the template recommendation. This requires us to further optimize the recommendation algorithm in the next step, so that the system can be more accurate. Make recommendations for the template.

Personalized recommendation technology was born in the field of e-commerce, and achieved a good recommendation [15]. The personalized recommendation system based on collaborative filtering is applied to the analysis of table tennis skills and tactics, which is of great significance for improving the analysis efficiency of coaches. With the development of the world table tennis level, the leading position of the Chinese Legion in this field is slowly facing the threat of countries such as Europe, America and Japan. How to maintain the dominance of China's table tennis in the world, this is the national team coach. And the new task of the athlete.
Scientific training and professional competition guidance are the key to achieving the above tasks, and the use of scientific and technological means to provide technical support to the coaches is a necessary condition for realizing scientific command competitions. The research in this paper has a positive effect on the improvement of scientific research level and helping to prepare for the 2020 Tokyo Olympic Games.

\section{CONFLICT OF INTEREST}

The authors declare no conflict of interest.

\section{AUTHOR CONTRIBUTIONS}

Jing Sun, Haochen Luo prepared this article and studied the feasibility; Jing Sun, Haochen Luo, and Huiqun Zhao concuted the research, performed experimental operations, obtained experimental data. Jing Sun and Haochen Luo concuted the research; Haochen Luo, Huiqun Zhao wrote the paper; all authors had approved the final version.

\section{REFERENCES}

[1] H. Zhao and J. Sun, "Sports Computing: A new field of computer application research," Computer Science, 2004, vol. 31, no. 8, pp. 89-92.

[2] S. Hu, D. Xiao, B. Feng, and X. Mi, "Development and application of table tennis technical and tactical analysis statistical software (key 2.0)," China Sports Technology, vol. 49, no. 4, pp. 37-42, 2013.

[3] H. Xu, W. Wu, and X. Li, "Comparative study of internet recommendation system," Journal of Software, vol. 20, no. 2, pp. 305-362, 2009.

[4] D. Bokde, S. Girase, and D. Mukhopadhyay, "Matrix factorization model in collaborative filtering algorithms: A survey," Procedia Computer Science, vol. 49, no. 4, pp. 136-146, 2015.

[5] G. Wang and H. Liu, "An overview of personalized recommendation system," Computer Engineering and Applications, vol. 2012, no. 7, pp. 66-76.

[6] J. Wang, C. Man, Y. Zhao, and F. Wang, "An answer recommendation algorithm for medical community question answering systems," in Proc. IEEE International Conference on Service Operations and Logistics, and Informatics, 2016, pp. 139-144.

[7] N. Kurmashov, K. Latuta, and A. Nussipbekov, "Online book recommendation system," in Proc. International Conference on Computational Intelligence and Computing Research. Almaty, Kazakhstan, 2015, pp. 1-5.

[8] H. Ma, X. Wang, and J. Hou, "Course recommendation based on semantic similarity analysis," in Proc. 2017 IEEE 3rd International Conference on Control Science and Systems Engineering, Wuhan, China, 2017, pp. 638-641.

[9] Y. Sun, T. Wang, Y. Huang, and Y. Sun, "Personalized recommendation system for Sina MicroComic users," Wireless Telecommunications Symposium. Chicago, USA, 2017, pp. 1-5.

[10] F. Sanchez, M. Alduan, F. Alvarez, J. M. Menendez, and O. Baez, "Recommender system for sport videos based on user audiovisual consumption," IEEE Transactions on Multimedia, pp. 1546-1556, 2012.

[11] A. K. Pandey and D. S. Rajpoot, "Resolving cold start problem in recommendation system using demographic approach," in Proc. International Conference on Signal Processing and Communication. Noida, India, 2016, pp. 213-218.

[12] S. Fan, H. Yu, and H. Huang, "An improved collaborative filtering recommendation algorithm based on reliability," in Proc. 2018 IEEE 3rd International Conference on Cloud Computing and Big Data Analysis, Chengdu, China, 2018, pp. 45-51.

[13] X. Zhang, D. Xiao, X. Zhou, and W. Fang, "Construction and application of three-segment statistical method for table tennis tactics and dynamics," China Sports Science and Technology, vol. 2018, no. 1, pp. 80-83.

[14] F. Wu, G. Liu, and C. Hua, "Study on improving technical and tactical statistical methods in table tennis 3," China Sports Technology, vol. 50, no. 1, pp. 71-74, 2014. 
[15] B. Yang and P. Zhao, "Review of Recommended Algorithms," Journal of Shanxi University, vol. 2011, no. 3, pp. 337-350.

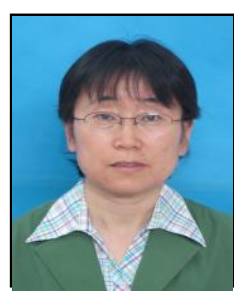

Jing Sun was born in January 1968 in Tieling City, Liaoning Province, China. From September 1986 to July 7 , she graduated from the Mathematics Department of Liaoning University with a Bachelor of Science degree. From September 1998 to July 2000, she graduated from the Department of Computer Science and Technology of Liaoning University with a master of science degree.

Her research interests are big data analytics, internet of things technology, and software engineering. She has participated in four projects of the National Natural Science Foundation of China and one of the Beijing Academic Innovation Team Projects. She also published more than 50 academic papers.

She is a senior member of the Chinese Computer Society.

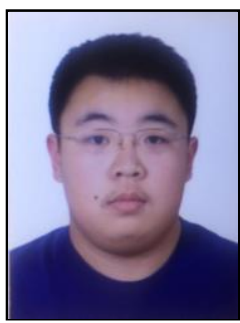

Haochen Luo was born in Beijing, China in May 1994. From September 2012 to 2016, Haochen Luo received a bachelor of computer science degree from the School of Computer Science and Technology of Beijing Information Science and Technology University. From August 2016 to Now, Haochen Luo studied master's degree in software engineering at North China University of Technology
During university studies, he participated in the National Table Tennis Olympic Games project under the guidance of Prof. Zhao Huiqun.

During he studies at the university, he received many awards for learning contests and research activities.

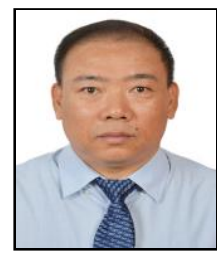

Huiqun Zhao was born in September 1960 in Anshan City, Liaoning Province, China. From September 1979 to July 1983, he obtained a bachelor of science degree in mathematics from Jinzhou Normal University. From September 1988 to March 1991, he obtained a master's degree in engineering from the Computer Science Department of Northeastern Institute of Technology. In July 2002, he studied in the Department of Computer Science and Engineering of Northeastern University and obtained a doctorate in engineering.

His research interests are big data analytics, IoT technology and software engineering. He has presided over four projects of the National Natural Science Foundation of China, one project of Beijing Natural Science Foundation, and one project of Beijing Academic Innovation Team. Published more than 100 academic papers.

$\mathrm{He}$ is a senior member of the Chinese Computer Society, a member of the software engineering committee of the Chinese computer society, the service computing professional committee, and the professional committee for fault tolerance. 